\title{
Deadline Probing: Towards Timely Cognitive Wireless Network
}

\author{
Panlong Yang^, Guihai Chen, and Qihui Wu \\ Institute of Communication Engineering, PLAUST \\ Computer Science Department, Nanjing University. Nanjing, Jiangsu Province, China \\ plyang@computer.org, gchen@nju.edu.cn, qiwu@google.com
}

\begin{abstract}
A confidential and effective probing is fundamental to a cooperative and cognitive wireless network. Previous seminar works are not deadline sensitive, and often suffer from highly dynamic multi-channel environments. As they focus more on transmitting packets with the optimal channel, resources are not efficiently used when sufficient channels are available in multi-radio multi-channel systems. Decisions are made without time constraints, while in dynamic wireless environments, deadlines are always presented for both probing and data transmission process. In this paper, we propose a transmission deadline probing paradigm, and an optimal probing and transmission schedule with time constraints is proposed, which is a pure threshold policy. Simulation results show that, deadline probing paradigm effectively improves network resource utilization as multiple channels presented with probing and transmission deadlines.
\end{abstract}

Keywords: Cognitive Radio, Mobility, Scheduling, Wireless Network.

\section{Introduction}

As fundamental resources in self-organizing wireless network, channels qualities and link durations are important factors in achieving efficient data transmissions. On one hand, mobility causes dynamic topology changes between mobile devices in network, which would eventually lead to packet loss and additional routing messages. Routing protocol overhead and undelivered roaming packets degrade network performance dramatically. On the other hand, spatial and temporal varying characteristics of wireless channels would make the probing policy difficult and degrade scheduling efficiency. Great efforts are needed in finding an opportunistically optimal channel and time period to transmit packets [1] [2].

At the same time, many research works [10] [5] have been done on selecting routes in highly dynamic and self-organizing wireless network. However, these works suffer from the following two aspects. One is that, although joint works

\footnotetext{
* This work is supported in part by the National Basic Research Program of China (973 Program) under grant No. 2006CB303004. China 863 project Grant No.2007AA01Z267. Jiangsu High-Tech Research Project of China under Grant No.BG2007391.
}

J. Cao et al. (Eds.): NPC 2008, LNCS 5245, pp. 38 49. 2008.

(C) IFIP International Federation for Information Processing 2008 
on routing, channel assignment and scheduling have been proposed, they are all based on a relative static network topology. For those algorithms based on globally optimizations, any changes on network topology would cause large amount of control messages overhead, which would degrade network performance as most of the time are spent on computing the optimal value and the optimal schedule. The other is that, unstable forwarder would cause intermittent links in network, which lead to frequent re-routing in network scale.

Deadline probing mechanism would intelligently select links with relatively long duration, and provide scheduling algorithm with time constraints. Scheduling according to both transmitting jobs and channel transmitting capability will make a more efficient usage on channels, and time constraints strengthen the scheduling algorithm in time scale.

The main contributions of this paper are listed as follows: Firstly, we propose an effective deadline probing algorithm in dealing with mobile wireless network environment. Link duration is the time constraints between two nodes, and by using the adaptive beacon messages, we explore the mobility factors of the moving node, and evaluate the link duration time, which is important to optimal channel assignment.

Secondly, we propose a multi-channel transmission schedule algorithm which considers traffic arrival rate and transmission deadline. Channels are assigned to the appropriate transmissions which is "suitable" to accomplish the transmitting job.

The remainders of this paper are organized as follows. We review the seminar works related in Section 2. Section 3 we formulate our problems. In section 4, we propose a deadline probing algorithm. Section 5 transmission schedule is modeled as a sequential job assignment algorithm with deadline constraints. Numerical results and simulation results are presented in section 6 . Section 7 concludes the paper.

\section{Related Work}

Many seminar works have been done in order to opportunistically utilize the multiple available channels [1] 2, 4] with relative good quality. In 4], Ji et al propose a scheme named Medium Access Diversity (MAD) to probe channel with time and space varying quality at the MAC layer. By effectively using the multi-channel diversity, the user can select the receiver with best channel condition. Channel probing with opportunistic transmission has been widely studied recently. Similar problems have been studied in [1] [2] [4.

In all those applications, optimal channel probing can be achieved in order to set a tradeoff between obtaining useful channel information and consuming valuable probing times. It is usually assumed in these applications that, more channels are ready for transmission, and the only thing probing policy needs to consider is to select the best one among them, with a joint considerations on probing cost. In multi-channel systems, independent optimization on one channel transmission is not sufficient. In the perspective view of overall network 
throughput, if wireless channels are enough, selecting one channel for transmission would reduce overall channel utilization.

Seminar work in [1] presents a pure threshold based algorithm on optimal stopping strategy of channel probing. It is a tradeoff between channel quality reward and probing cost. 2] propose a distributed scheme, which models the probing procedure as a contention and threshold based process. Seminar work in 1 assumes that channel state is independent of the state of other channels during transmission. It is true if we take channel state as the only metric for opportunistic transmission. However, in real network deployment, if the channels are not enough, the links in network would congest for limited number of channels. Selecting one channel would face challenges, one is that the larger reward channels would possibly be preferred by all nodes, and the correlations between channels 1] would somehow make the contention even worse. Another challenge is that, as the time progresses, more channels are probed, and the rest of the channels are unused by other transmissions are becoming less, which would the contention on channel selection.

These works also assume that channel sates are rather stable in a relative long period, but it is not true since channel quality is a parameter varying with time [5] 6].

\section{Problem Formation}

\subsection{Deadline Probing Model}

The movement model of each node in network is random way point (RWP), where each node in network randomly selects a position as its destination in a convex region, and selects a speed randomly with a uniformly distributed region $\left[v_{\min }, v_{\max }\right]$, and the node moves toward its destination at its chosen speed directly. Transmission power can be adaptively adjusted in the transmission power set $\Pi=\left\{P_{1}, P_{2}, \ldots, P_{m}\right\}$. There are $m$ levels of transmission range respectively, which form the range set $\Gamma=\left\{R_{1}, R_{2}, \ldots, R_{m}\right\}$. At the physical layer, we simplified our model to free-space model and a SINR based receiving, as defined in 11; while at the MAC layer, we use the IEEE 802.11 model. As mentioned in the following sections, our proposed scheduling and mobility awareness mechanism are independent to MAC layer protocols, and only need a power adjustable transmission scheme.

In mobile wireless network, duration time between nodes in dynamic topology is hard to achieve. The first problem is that in highly dynamic network, transit links are always existing in network entirely. These links would lead network communication into fluctuation state, and no effective mobility mining is available as links in network are unstable. The second reason is that, nodes are moving in different velocity, with different speed and direction. As RSSI estimation methods have been applied [10, it will suffer from weak and unstable radio signals. 
In order to solve these problems, we need a mobility-aware mechanism in evaluating the link stability. And we assume that, no localization and speed measurement instrument is available to nodes in network.

\subsection{Channel Quality Probing and Scheduling}

We consider a wireless system consisting of $n$ channels. There are $\mathcal{N}$ nodes in graph $\mathcal{G}=\langle\mathcal{V}, \mathcal{E}\rangle$, with $\mathcal{M}$ edges such that $e(i, j)=\left\langle v_{i}, v_{j}\right\rangle \in \mathcal{E}$. With each channel $j \in \Omega$, the channel quality would be a random variable $X_{j}$. It is assumed that, each channel must be probed before being used for transmission after a period of that in order to avoid the channel quality variation 6] 5 . We also assume that, channel quality values are independently and identically distributed if homogeneous network is given. While in a heterogeneous network, distribution of different channels varies.

As we use multiple channels to transmit packets dynamically and concurrently, the top $k$ among all channels having been probed are selected in terms of their channel quality value $X_{j}$, which could be modeled as the following equations:

$$
\operatorname{Max} E\left[J_{N}\left(\boldsymbol{u}_{1}^{N}\right)\right]=E \sum_{1 \leq k \leq N} u_{k} X_{k}
$$

with $\boldsymbol{u}_{1}^{N}=\left(u_{1}, u_{2}, \ldots, u_{N}\right)$ satisfying the constraints. $u_{j} \in \mathcal{U}_{j}=\{0,1\}, j=$ $1,2, \ldots, N, \sum_{1 \leq k \leq N} u_{k}=n . J_{N}(*)$ denotes the reward value, in this paper, it denotes the probed channel bandwidth. $E[*]$ denotes the expectation of reward value.It has been proved in [7] that, at each step $j$, with $N-j+1$ channels still available and $a_{j}$ already selected for transmission. And the optimal result is up to a threshold value $\tilde{s}\left(j, a_{j}\right)$ such that the channel $j$ with quality value $X_{j}$ is selected if larger than $\tilde{s}\left(j, a_{j}\right)$ and is rejected otherwise. Although such threshold probing criteria is optimal for multi-channel probing problem, it does not consider limitations on channel probing duration and number of admissible channels.

As multiple channels can be probed, another question naturally arises that, as the packets for transmission arrive sequentially, locally assign packets to different channels would be important, as packets should be successfully transmitted before the probing deadline reaches, and not all transmission need to be transmitted on optimal channels. And the local channel assignment algorithm can be described as:

Problem. How to assign sequentially arriving packets to multiple channels with different bandwidth in order to maximize number of successful transmissions, where each packet transmission has a transmission deadline?

\section{Deadline Probing Algorithm}

\subsection{Threshold Based Filtering}

As shown in Fig. 1, node $i$ and node $j$ are located on position in planar labeled $O$ and $P$ respectively. According to deadline probing algorithm, probing range 


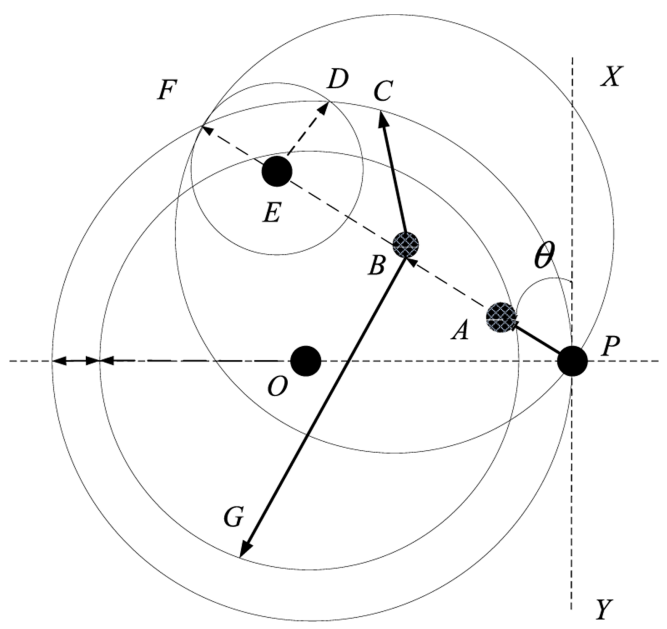

Fig. 1. Threshold based neighbor selection

is $\|\overrightarrow{O P}\|=R$, with maximum transmitting power. And the one-phase tolerant transmission range is $\|\overrightarrow{O Q}\|=(1-\Delta) R$. And we denote $\Delta$ as "tolerance factor". Node $j$ is moving along direction at $\overrightarrow{B P}$. We assume that, $\angle B P X=\phi$. Since $\begin{aligned}\|\overrightarrow{B N}\| & =\|B P\|-\|P N\|=2\|P M\|-(\|P M\|-\|M N\|) \\ & =\|P M\|+\|M N\|\end{aligned}$
$\|\overrightarrow{P M}\|=R \cdot \sin \theta,\|\overrightarrow{M N}\|=\sqrt{(1-\Delta)^{2} R^{2}-(R \cos \theta)^{2}}$.

And it can be concluded that,

$$
\|\overrightarrow{B N}\|=R \cdot \sin \theta+\sqrt{(1-\Delta)^{2} R^{2}-(R \cos \theta)^{2}}
$$

Available link duration is denoted as $U(\theta, v, \Delta)$. Threshold based stability means that link duration $\|\overrightarrow{B N}\| \geq U_{\text {thre }}$ and we can get the $\theta_{\min }$ and $\theta_{\max }$, and accordingly the tolerant factor $\Delta$. According to series of equations listed above, we can get the value that, $\theta_{\min }=\arcsin \frac{U_{\text {thre }}}{R}$ and $\theta_{\max }=\pi-\arcsin \frac{U_{\text {thre }}}{R}$.

And the reduction factor

$$
\Delta=1-\sqrt{1-\left(U_{\text {thre } / R}\right)^{2}}
$$

There are two factors that are not available: direction and velocity, which affect the link stability most in random way point model. In the following subsection, we will make an investigation on direction and velocity awareness on random way point mobility model. 


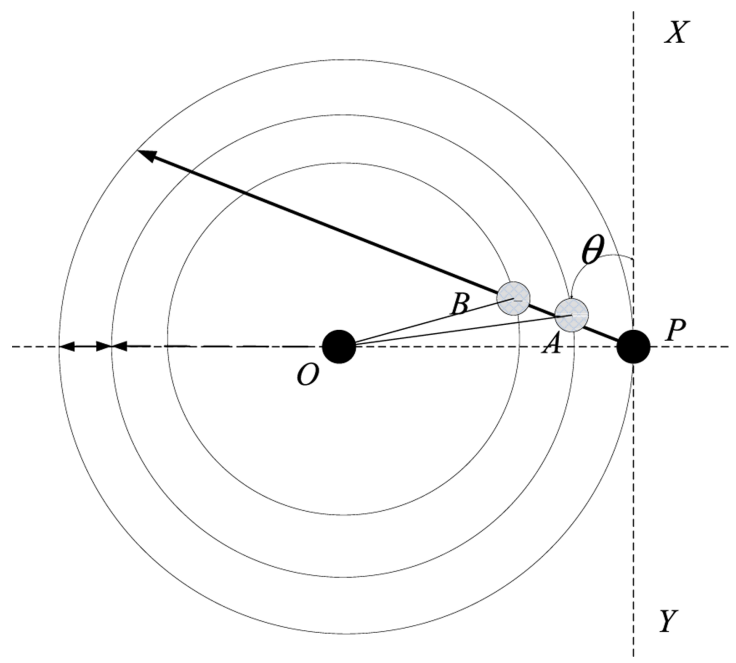

Fig. 2. Direction and velocity awareness mechanism

\subsection{Relative Direction and Velocity Awareness}

Threshold based filtering can effectively filter out the transitory links with duration below, but the velocity is not available, and the direction is not available also. In this subsection, we assume the centering node is static, and the relative direction and velocity can be achieved according to the following equations. As shown in Fig. 2, we can have that

$$
\begin{aligned}
& \|P A\|=v \cdot t_{1}=R \cdot \sin \theta-\sqrt{\left(1-\Delta_{1}\right)^{2} R^{2}-(R \cos \theta)^{2}} \\
& \|P B\|=v \cdot t_{2}=R \cdot \sin \theta-\sqrt{\left(1-\Delta_{2}\right)^{2} R^{2}-(R \cos \theta)^{2}}
\end{aligned}
$$

$\|P A\|=\frac{t_{1}}{t_{2}}$ and we can get an equation that:

$$
t_{1} \cdot\left[\sin \theta-\sqrt{\left(1-\Delta_{1}\right)^{2}-\cos ^{2} \theta}\right]=t_{2} \cdot\left[\sin \theta-\sqrt{\left(1-\Delta_{2}\right)^{2}-\cos ^{2} \theta}\right]
$$

Obviously, if $\Delta_{1}=\Delta_{2}$, then $t_{1}=t_{2}$. If we intentionally choose $\Delta_{1} \neq \Delta_{2}$, we can calculate parameter $\theta$. Let left $f(\theta)$ denote left part of equation 6 , and let $g(\theta)$ denote right part of equation 6 .

$$
\begin{aligned}
& f(\theta)=\sin \theta-\sqrt{\left(1-\Delta_{1}\right)^{2}-\cos ^{2} \theta} \text { and } g(\theta)=\sin \theta-\sqrt{\left(1-\Delta_{2}\right)^{2}-\cos ^{2} \theta} \\
& \frac{f\left(\frac{\pi}{2}\right)}{g\left(\frac{\pi}{2}\right)}=\frac{1-\sqrt{\left(1-\Delta_{1}\right)^{2}}}{1-\sqrt{\left(1-\Delta_{2}\right)^{2}}}=\frac{\Delta_{1}}{\Delta_{2}}
\end{aligned}
$$


As $\theta=\frac{p i}{2}$, we have

$$
\frac{f\left(\frac{\pi}{2}\right)}{g\left(\frac{\pi}{2}\right)}=\frac{1-\sqrt{\left(1-\Delta_{1}\right)^{2}}}{1-\sqrt{\left(1-\Delta_{2}\right)^{2}}}=\frac{\Delta_{1}}{\Delta_{2}}
$$

As $\theta=\arccos \left(1-\Delta_{2}\right)$

$\frac{f\left(\arccos \left(1-\Delta_{2}\right)\right)}{g\left(\arccos \left(1-\Delta_{2}\right)\right)}=\frac{\sqrt{1-\left(1-\Delta_{2}\right)^{2}}-\sqrt{\left(1-\Delta_{1}\right)^{2}-\left(1-\Delta_{2}\right)^{2}}}{\sqrt{1-\left(1-\Delta_{2}\right)^{2}}}$ And we have $d\left[\frac{f(x)}{g(x)}\right] / d x<0$, thus the function is monotonic.

As figure Fig 2 shows, two phase probing improves the performance of deadline probing algorithm, where the mobile direction and speed if we set different tolerant factors in two phase probing process. According to our deadline probing paradigm, both direction and speed of the moving node can be evaluated through adaptive power adjustment and probing mechanism.

\section{Channel Assignment with Job Complete Deadline}

\subsection{Model Description}

In our channel assignment model, arriving packets should be assigned immediately or rejected. Either there is a single deadline that is exponentially distributed with rate $\alpha$, or there are $n$ independent deadlines exponentially distributed. Packets being rejected for transmission should be buffered and re-scheduled so as to to be transmitted on next probing period. In this paper, we aim at maximizing the probability that at least $k$ transmitting jobs out of $n$ are correctly completed before the deadline. On each channel $j$ of node $v_{i}$, there is a competency value $p_{j}$, which is defined as follows.

Definition 1: Competency value $p_{j}$ for channel $j$ is the probability that a metric on channel quality, it can be the successful transmission rate or a normalized bandwidth, where $0 \leq p_{j} \leq 1$ probed according to threshold based strategy.

Each channel $j \in \Omega$ would have competency value $p_{j}$, and transmit data on the channel $j$ with quality $X_{j}$. The is channel $j$ can be correctly used link $i$ with rewards $p_{i} X_{j}$. The channel quality can be achieved through probing, and appealing to renewal theory, the channel is available for transmission can be modeled as a queuing system, where the arrival rate can be applied in characterizing channel availability for transmission.

According to sequential assignment problem studied in [8, we have the following theorem in achieving maximized number of success transmissions. There are the following characteristics of our problem:

1: The channel resource is limited; 2 : Request arrival rate for the resource is a stochastic process; 3: The rewards associated with the channel resource is known before transmission; 4: A deadline exists, where unfinished transmissions would be rejected from transmission; 5: Reject or accept the transmission should be decided on-line; 6 : The objective is to maximize the expected reward accumulated by the deadline. 
Let us consider the link competency values so that $p_{1} \geq p_{2} \geq p_{3}, \ldots, p_{n} \geq 0$. Let $X$ be a random value on success probability of packet transmission. Obviously, $0 \leq X \leq 1$, and it is dominated by packet arrival time and packet transmission time. The problem is how to assign $m$ different packet transmissions to $n$ channels, which would achieve a maximized number of success transmissions.

The optimal assignment policy can be modeled as:

$$
\begin{gathered}
\operatorname{Max} \quad u_{i j} p_{i} x_{j} \\
\text { subject to } \quad p_{i} \in\{0,1\} \quad x_{j} \in\{0,1\} \quad u_{i j} \in\{0,1\} \\
i \in\{1, \ldots n\} \quad j \in\{1, \ldots, m\} \\
\sum_{i=1}^{n} u_{i j} \leq 1 \quad \sum_{j=1}^{m} u_{i j} \leq 1
\end{gathered}
$$

If packet numbered $i$ is assigned to channel $j, u_{i j}$ would accordingly be set to 1 , else it would be 0 .

\subsection{General Results}

If there are no limits on number of packets waiting for transmissions, and the deadlines for each packet are independent, we will get the following theorem $[8]$.

Theorem 1. Given an optimal assignment policy, there are thresholds values on transmitting job difficulty, denoted as $1=v_{0}>v_{1}>v_{2}, \ldots, v_{n}>v_{n+1}=0$, such that to assign transmission job value $x$ to channel $i$ if $v_{i}<x \leq v_{i-1}$.

THEOREM 1 means that, channel assignment policy can be reduced to pure threshold policy, where channel assignment is done according to the rank of the "job difficulty", and it shows to us that, the optimal assignment policy is a pure threshold based policy.

Lemma 1. Let $\pi$ denotes assignment policy and the optimal threshold corresponding to policy $\pi$ can be computed according to the following iterative equation.

$$
v_{i}=\frac{\lambda P\left[X>v_{i}\right]}{\lambda P\left[X>v_{i}\right]+i \alpha} E\left[X \mid X>v_{i}\right]
$$

Corollary 1: If the transmission deadline parameter on channel with best transmission quality is $i \cdot \alpha$, and have the same deadline threshold as the $i^{t h}$ arrival packet, that is the deadline parameter of the first arrival packet is $i \cdot \alpha$, it will have the same threshold as the $i^{\text {th }}$ channel has the deadline parameter of $\alpha$, because $v_{1}(i \cdot \alpha)=v_{i}(\alpha)$. 


\subsection{Reward on Transmitting Packets}

In this paper, we denote the random value $x$, as the transmitting reward $x_{i}=$ $P\left[F_{i}=T_{i}+A_{i}<D_{i}\right]$, where $T_{i}$ is the transmission duration of packet labeled with $i$, and $T_{i}=\frac{l_{i}}{b} . A_{i}$ is the arrival time of the packet, and the ending time of a packet can be denoted as $F_{i}=T_{i}+D_{i}$. And $D_{i}$ is the delay constraint. A question naturally arises, that is, how to set the bandwidth value $b$ ? It is shown in [11] 6] that, channel bandwidth is correlated with channel quality, and $T_{i}=\frac{l_{i}}{b_{j}}$. One simple method is that, each reward value $x$ is evaluated equally with best channel quality $\sup _{\left\{p_{j} \in \mathcal{P}\right\}}\left\{p_{j}\right\}$. Under this policy, packets with shorter length would be preferred over longer ones, and more likely to be transferred on channels with higher quality. Because according to this criteria, shorter packets would have larger $x$, and shorter packets are more likely to be transmitted on high quality channels.

Considering network throughput, each packet should be transmitted on its suitable transmission channel. That is, if the packet has larger length, it should be transmitted on high quality channel.

And according to this lemma, we can easily get the following results. If the packet labeled $i$ is accepted for transmission, and let $l_{i}$ denote the packet length, $t(i)_{j}=\frac{l_{i}}{b_{j}}$ denote the transmission time value of packet $i . x(i)_{j}$ is monotonic with the order of channel $j$. As $1=v_{0}>v_{1}>v_{2}, \ldots, v_{n}>v_{n+1}=0$, with increasing of $j, x(i)_{j}$ decreases. There are three possible results as we select channel $j$ to transmit packet $i$. They are $j^{*}>j, j^{*}=j$ and $j^{*}<j$ respectively.

Lemma 2. If a transmission can be accepted for transmission, suitable channel $i^{*}$ exists, and the value is $x\left(i^{*}\right)$.

Proof. There are three cases as we arbitrarily select one channel for transmission. The first case is $x<v_{i}$; The second case $x>v_{i-1}$; And the third case is $v i<$ $x<v_{i-1} . x(i)$ is non-increasing as $i$ increases. In the third case, it proves that, channel $i=i^{*}$. In the first case, we should decrease $i$, whereas, the reward value $x$ would increase. Since the transmission can be accepted, there is $0 \geq i^{*}<i$, and $v i^{*}<x<v_{i^{*}-1}$. In the second case, we should increase $i$, where the reward value is decreased accordingly.

Since threshold values are monotonic, our suitable channel finding algorithm (SCF) works as follows:

Step 1: Compute $x$ according to $P\left[F_{i}=T_{i}+A_{i}<D_{i}\right]$ when channel $i$ is used for transmission

Step 2: if $x<v_{i}, i=i-1$, go to step 1 ;

if $x>v_{i-1}$ then $i=i+1$, go to step 1 ;

if $v i<x<v_{i-1}$, end the algorithm.

At first, the transmission job $T_{i}$ randomly select a channel $j$ and evaluate the transmission difficulty $x$, if $v_{j}<x(i) \leq v_{j-1}$, the transmission channel would be $j$. Else if $x(i) \leq v_{j}$, we should select channel $j-1$, if . Else if $x(i)>v_{j}$, we should select channel $j+1$. 


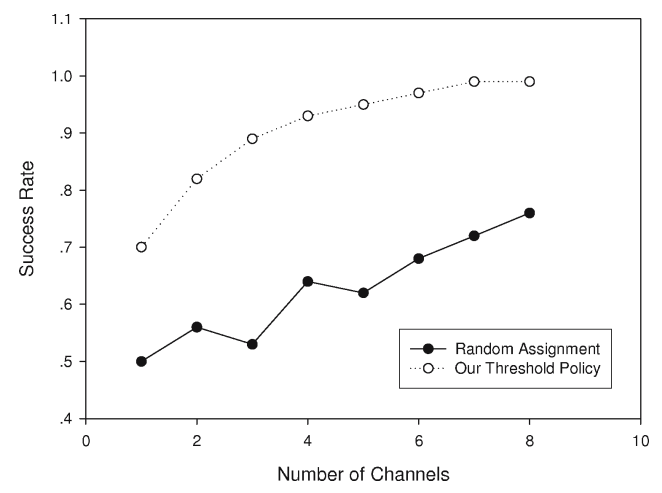

Fig. 3. Different transmission schedule comparisons

\subsection{Sub-optimal Criteria}

Iteratively computing the threshold value would be difficult as distribution of job difficulty is complex. At the same time, we are also interested on achieving at least $k$ successful transmissions with maximal probability.

In this subsection, we first considering a special case where there only two channels available, and the object is to maximize probability that at least one packet is successfully transmitted over the two channels. Assume that there are two channels and $p_{1} \geq p_{2}$, and the deadline is exponentially distributed with rate $\alpha$. The theorem has been proved in [8] as follows:

Theorem 2. The optimal policy is to assign a channel with value $x$ to link-set 1 if $x>v_{1}$, to assign it to link-set 2 , if $v_{1} \geq x>t$, and to reject it otherwise, where

$$
\begin{gathered}
v_{1}=\frac{\lambda q_{1} E\left[X_{1}\right]}{\alpha+\lambda q_{1}} \\
t=\frac{\lambda q_{1} v_{1}\left(1-p_{1} E\left[X_{1}\right]\right)}{\left(\lambda q_{1}+\lambda q_{2}+\alpha\right)\left(1-p_{1} v_{1}\right)}+\frac{\lambda q_{2} E\left[X_{2}\right]}{\lambda q_{1}+\lambda q_{2}+\alpha}
\end{gathered}
$$

Also, $V\left(p_{1}, p_{2}\right)=p_{1} v_{1}+\left(1-p_{1} v_{1}\right) p_{2} t$

Comparing with the policy that try to maximized number of successful transmissions, the first threshold $v_{1}$ in both cases is the same, since there is only one channel to be assigned, and it is independent to the second channel. The second threshold $t>v_{2}$, as the object that we set threshold value $t$ is to maximize the probability of at least one transmission is successful.

Let $V_{k, n}(\boldsymbol{p} \mid x)$ be the value of $P\{N \geq k\}$ under policy $\pi$ when there are $n$ links with competency value array $\boldsymbol{p}$, and the channel with reward $x$ has just arrived.

$V_{k, n}(\boldsymbol{p})=p_{n} v_{n} V_{k-1, n-1}\left(\boldsymbol{p}^{n}\right)+\left(1-p_{n} v_{n}\right) V_{k, n-1}\left(\boldsymbol{p}^{n}\right)$ for all $n>0,0<k \leq n$.

$V_{0, n}(\boldsymbol{p})=1$ for all $n$,

$V_{k, n}(\boldsymbol{p})=0$ for all $k>n$. 
We find that, the $k$ out of $n$ optimal criteria and maximum number criteria are correlated with channel states and the distribution of transmission jobs. We will make a further discussion in our simulation works.

\section{Simulation Results and Numerical Analysis}

We build the simulator and it is written in $\mathrm{C}++$ language. In this simulation, we build a 10 nodes network, where channel reward value are uniformly distributed between $(0.7,0.9)$, deadline parameter $\alpha$ is uniformly distributed between $(0,4)$. Packet length is triangular distributed with average packet length $E[L]=5$. Appealing to free-space radio propagation model and SINR based receiving [1], we set our simulation environment, where the transmission range is $250 \mathrm{~m}$.

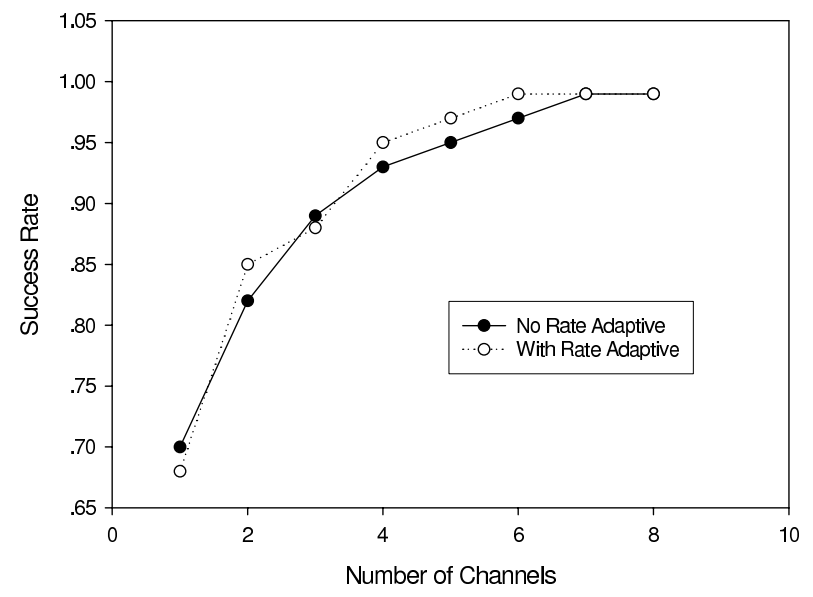

Fig. 4. Comparisons on rate adaptive mechanism with success rate

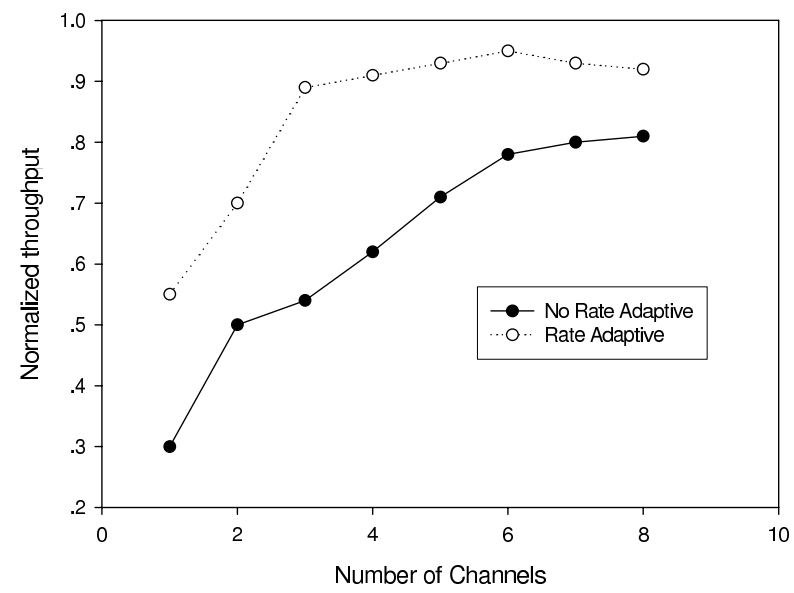

Fig. 5. Comparisons on rate adaptive mechanism with normalized throughput 
In Fig3, we make a comparison on success probability between random assignment policy and our threshold based policy.

In Fig4, we make an evaluation on rate adaptive algorithm with neighbor coordination, which is shown in section4.

Simulation show that although rate adaptive algorithm does not improve success rate obviously, it really improves network throughput due to its consideration on packet length, which is shown in Fig 5 .

\section{Conclusions}

In this paper, we model optimal probing problem as a knapsack problem with deadlines, and channels probed are sequentially accepted or rejected according to threshold value. Transmission schedule policy is built according to pure threshold criteria, where transmission jobs are assigned to different channels, leading to a maximized number of successful transmissions as deadlines are considered as well. Future work will include a more accurate model on channel reward values and transmission priority. Quantum of different channels should be fully considered, which will affect channel utilization in entire network scale.

\section{References}

1. Chang, N.B., Liu, M.: Optimal channel probing and transmission scheduling for opportunistic spectrum access. In: ACM Mobicom, September 2007, pp. 795-825 (2007)

2. Zheng, D., Ge, W., Zhang, J.: Distributed opportunistic scheduling for ad-hoc communications: an optimal stopping approach. In: ACM Mobihoc, pp. 795-825 (2007)

3. Guha, S., Munagala, K., Sarkar, S.: Jointly optimal transmission and probing strategies for multichannel wireless systems. In: 40th Annual Conference on Information Sciences and Systems, pp. 955-960 (2007)

4. Ji, Z., Yang, Y., Zhou, J., Takai, M., Bagrodia, R.: Exploiting medium access diversity in rate adaptive wireless lans. In: Proc. of IEEE Asilomar Conference on Signals, Systems, and Computers (September 2004)

5. Wang, H., Mandayam: Opportunistic file transfer over a fading channel under energy and delay constraints. IEEE Transactions on Communications, 632-644 (2005)

6. Wu, D., Negi, R.: A Wireless Channel Model For Support of Quality of Service. IEEE Transactions on Wireless Communications, 630-643 (2003)

7. Derman, C., Lieberman, G., Ross, S.: A Sequential Stochastic Assignment Algorithm. Management Science, 349-355 (1972)

8. Righter, R.: Stochastically Maximizing the Number of Successes in a Sequential Assignment Problem. Journal of Applied Probability, 351-364 (1990)

9. Gunter, Stefan, G., et al.: Queueing Networks and Markov Chains. Publishing House of Electronics Industry (1998)

10. Lenders, V., Baumann, R.: Link-diversity Routing: A Robust Routing Paradigm for Mobile Ad Hoc Networks. Technical Report (2006)

11. Rappaport, T.S.: Wireless Communication: Principles and Practice. Prentice Hall, Englewood Cliffs (1995) 